\title{
O papel de mediação das necessidades psicológicas na associação entre o suporte de autonomia e o bem-estar psicológico em praticantes de fitness
}

\author{
The mediating role of psychological needs in the association between the \\ autonomy support and psychological well-being in fitness exercisers
}

João Moutão ${ }^{1,2 *}$, Susana Mendes Alves ${ }^{1,3}$, Diogo Monteiro ${ }^{1,2}$, Luís Cid ${ }^{1,2}$

ARTIGO ORIGINAL | ORIGINAL ARTICLE

\begin{abstract}
Tendo por base a teoria da autodeterminação, este estudo objetiva a análise do papel mediador da perceção de satisfação das necessidades psicológicas básicas na relação entre a perceção de suporte de autonomia dado pelo instrutor de fitness e indicadores de bem-estar psicológico, em praticantes de exercício. Participaram neste estudo 984 praticantes de fitness em ginásios, de ambos os géneros (531 femininos; 453 masculinos), com idades compreendidas entre os 16 e os 70 anos $(M=31.7 ; S D=11.2)$. Todos os praticantes foram avaliados ao nível da sua perceção de suporte de autonomia dado pelo instrutor de fitness, perceção de satisfação das necessidades psicológicas básicas (competência, autonomia e relação) e bem-estar psicológico (vitalidade subjetiva, satisfação com a vida e autoestima global). Os resultados obtidos, com recurso à análise de equações estruturais, corroboram o papel mediador das necessidades psicológicas básicas na relação entre a perceção de suporte de autonomia e o bem-estar psicológico. Estes resultados clarificam o mecanismo de promoção do bem-estar psicológico em praticantes de exercício, auxiliando no processo de delineamento de intervenções futuras no contexto do fitness com este propósito.
\end{abstract}

Palavras-chave: teoria da autodeterminação, motivação, instrutor de fitness, ginásio

ABSTRACT

In accordance with the tenets of self-determination theory, this study aims to examine the mediating role of basic psychological needs satisfaction in the relationship between perception on fitness instructor's autonomy support and psychological well-being indicators, among Portuguese exercise participants at the context level of generality. Participate in this study 984 fitness exercisers, of both genders (531 feminine; 453 masculine) with ages between 16 and 70 years old $(M=31.7 ; S D=11.2)$, who completed measures assessing fitness instructor autonomy support perception, basic psychological need satisfaction (competence, autonomy and relatedness) and psychological well-being (subjective vitality, satisfaction with life and global self-esteem). Structural equation modeling analysis conducted support the partial mediating role of the of basic psychological needs satisfaction in the relationship between perceived autonomy support and the psychological well-being indicators. These results clarify the mechanism of psychological well-being promotion among fitness exercisers, helping in the future design of interventions in the fitness context with this purpose.

Key words: self-determination theory, motivation, fitness instructor, health clubs

Artigo recebido a 10-04-2014; Aceite a 24-09-2014

${ }^{1}$ Escola Superior de Desporto de Rio Maior (ESDRM-IPSANTAREM), IPS, Rio Maior, Portugal

${ }^{2}$ Centro de Investigação em Desporto, Saúde e Desenvolvimento Humano (CIDESD), Portugal

${ }^{3}$ Centro de Investigação em Qualidade de Vida (CIEQV), Portugal

* Autor correspondente: ESDRM-IPSANTAREM. Avenida Dr. Mário Soares, 2040-413 Rio Maior, Portugal. mail: jmoutao@esdrm.ipsantarem.pt 


\section{INTRODUÇÃO}

A teoria da autodeterminação (SDT) (Deci \& Ryan, 1985, 2008) é uma macro teoria motivacional que nos últimos anos tem conhecido um crescente interesse de aplicação ao contexto do exercício físico (Teixeira, Carraça, Markland, Silva, \& Ryan, 2012). De acordo com a SDT toda a atividade humana ocorre integrada num contexto social, seja ele real, por estarem fisicamente presentes outras pessoas, ou imaginado, por inconscientemente agirmos de forma como achamos que outras pessoas gostariam que nos comportássemos (Deci \& Ryan, 1985), sendo a qualidade desse "contexto social" que irá afetar o comportamento e o bem-estar dos indivíduos (Deci \& Ryan, 2008).

A SDT (Deci \& Ryan, 1985, 2008) explicita assim o mecanismo através do qual o efeito do ambiente social se faz sentir sobre o comportamento e o bem-estar psicológico dos indivíduos, o qual se concretiza por intermédio da satisfação das necessidades psicológicas básicas (NPB) de autonomia, competência e relação. A necessidade de autonomia representa a vontade de se ser a origem do próprio comportamento, distinguindo-se do conceito de "independência" ou de "liberdade total", refletindo antes uma "aceitação" e "envolvimento" num comportamento escolhido. A necessidade de competência reflete o desejo de interagir eficazmente com o meio envolvente, e é satisfeita quando se é bemsucedido na realização de tarefas e atividades desafiantes. A necessidade de relação espelha a aspiração de afiliação a outras pessoas ou a um determinado contexto social, e que traduz a necessidade de se desenvolver sentimentos de pertença. Estas necessidades são tidas como inatas e universais, quer isto dizer que não são aprendidas e são relevantes para $o$ comportamento humano independentemente do género, etnia ou repertório cultural, mesmo considerando que os meios para a sua satisfação possam diferir (Deci \& Ryan, 2008).

Desta forma, um ambiente social que suporte a autonomia dos indivíduos (e.g. fornecendo opções de escolha; dando um racional teórico; considerando a perspetiva dos outros; minimização da pressão) (Deci, Eghrari, Patrick, \& Leone, 1994), irá promover a perceção de satisfação das necessidades psicológicas básicas resultando em sentimentos de vitalidade e bem-estar. Um exemplo disso, é um instrutor de fitness que tem em consideração a perspetiva dos praticantes e incentiva-os a tomarem as suas próprias decisões, ao invés de impor o seu ponto de vista fazendo com que sejam os praticantes de exercício a terem de se adaptar às suas ideias (Edmunds, Duda, \& Ntoumanis, 2008).

Alguns estudos têm demonstrado o efeito positivo da satisfação das NPB sobre o bemestar (Edmunds et al., 2007), a autoestima global (Hein \& Hagger, 2007; Wilson \& Rodgers, 2002) e a vitalidade subjetiva (Vlachopoulos \& Karavani, 2009). O suporte de autonomia dado pelos instrutores de fitness tem também sido relacionado com a melhoria, no pós-exercício, da perceção de vitalidade e de estados de tranquilidade (Vlachopoulos \& Triantafyllidou, 2008). Importa também referir que esta resposta afetiva pós-exercício tem também um efeito retroativo sobre o comportamento, influenciando a decisão de voltar a praticar exercício, conforme têm comprovado alguns estudos recentemente realizados (Kiviniemi, Voss-Humke, \& Seifert, 2007; Williams et al., 2008). No caso específico do estudo realizado por Williams et al. (2008), verificou-se que a resposta afetiva ao exercício foi preditor da prática de exercício nos 6 e 12 meses seguintes. A promoção do bem-estar dos praticantes pode assim ser considerada como um fim ou também como um meio de promoção da própria prática de exercício, o que reforça ainda mais a importância da sua consideração neste contexto.

Os estudos analisados reforçam também o papel de relevo que os instrutores de fitness podem assumir na promoção do bem-estar e da adesão ao exercício, uma vez que são estes que orientam as sessões de exercício e que poderão em primeira instância suportar ou não a satisfação das NPB, demonstrando interesse, dedicando recursos, dando apoio e feedback constante, através do estabelecimento de numa relação de confiança e afetividade com os 
praticantes. Estes pressupostos têm sido testados também em Portugal para explicar as diferenças individuais de prática de exercício e de bem-estar (Silva et al., 2011; Silva et al., 2010; Teixeira et al., 2010; Vieira et al., 2011; Vieira et al., 2013). Todavia, os estudos referidos foram realizados no âmbito de programas institucionais de perda de peso, de livre acesso, e com recurso a alguns instrumentos de medida que não foram desenvolvidos especificamente para a avaliação das variáveis da SDT no contexto do exercício físico. Note-se que ao nível dos instrumentos de medida, Ryan (1995) refere que a investigação relacionada com a SDT deve ser realizada no âmbito de um contexto específico, apelando para o desenvolvimento e uso de questionários que tenham sido desenvolvidos especificamente para um determinado contexto de aplicação.

Um outro aspeto a ter em consideração nos estudos realizados em Portugal prende-se com o facto a maioria se centrar sobre o paradigma de relação bivariada de causa-efeito, não consideração dos efeitos de mediação, uma vez que como referem Baron e Kenny (1986), uma associação significativa entre uma variável independente e outra dependente é muitas vezes mediado por uma terceira variável que altera a magnitude e direção desta associação. Por essa razão, Teixeira et al. (2012), na mais recente revisão sobre a aplicação da SDT ao contexto da atividade física, reforça a importância de se conduzirem estudos futuros com análises estatísticas mais sofisticadas (e.g. análise de equações estruturais), para clarificar o papel mediador da satisfação das necessidades psicológicas básicas no âmbito da SDT.

Tomando em consideração os aspetos anteriormente mencionados, o presente estudo tem como objetivo analisar o papel mediador da perceção de satisfação das necessidades psicológicas básicas na relação entre a perceção de suporte de autonomia dado pelo instrutor de fitness e indicadores de bem-estar psicológico (i.e., satisfação com a vida, vitalidade subjetiva e autoestima global), com recurso a técnicas de estatística de análise de equações estruturais e à utilização de instrumentos de medida desenvolvidos especificamente para o contexto do exercício.

\section{MÉTODO}

\section{Amostra}

Participaram neste estudo 984 praticantes de fitness em ginásios, dos Distritos de Santarém e Leiria - Portugal, de ambos os géneros (531 femininos; 453 masculinos) e com idades compreendidas entre os 16 e os 70 anos $(M=$ 31.7; $S D=11.2)$. Os sujeitos auto relataram uma experiência de prática entre 1 a 480 meses $(M=45.5 ; S D=57.4 .1)$, uma frequência de prática entre 1 a 7 sessões por semana $(M=3.2$; $S D=1.2$ ), uma duração de prática semanal entre 1 a 15 horas $(M=5.0 ; S D=2.5)$ e um envolvimento em atividades de musculação (272), aulas de grupo (390) e cardiofitness combinação de atividades aeróbias e musculação (322). Como informação adicional podemos acrescentar ainda que 353 praticantes (36\%) frequentavam o ginásio, onde praticavam atualmente exercício, há menos de 6 meses, 271 praticantes $(28 \%)$ entre 6 e 18 meses, 229 praticantes (23\%) há mais de 18 e menos de 60 meses e 131 praticantes (13\%) há mais de 60 meses.

\section{Instrumentos}

Perceived Autonomy Support Exercise Climate Questionnaire - versão Portuguesa (PASECQp): consiste num instrumento de autorrelato adaptado ao contexto do exercício físico por Edmunds et al. (2006) a partir da versão original do Perceived Autonomy Support: Health Care Climate Questionnaire (Williams, Grow, Freedman, Ryan, \& Deci, 1996) tendo sido posteriormente traduzido e validado para a língua Portuguesa (Moutão, Cid, Leitão, \& Alves, 2012). Este questionário é constituído por 6 itens, que concorrem para um único fator que avalia a perceção do suporte de autonomia dado pelo instrutor de fitness (e.g. demostra confiança na minha capacidade de realizar os exercício). A resposta é dada numa escala ordinal de 1-7, correspondendo a opção "Discordo Totalmente" ao valor 1 e o "Concordo Totalmente" ao valor 7. No presente 
estudo este fator apresentou níveis elevados de consistência interna (alfa de Cronbach $=0.88$ ).

Basic Psychological Needs in Exercise Scale versão Portuguesa (BPNESP): consiste num instrumento de autorrelato desenvolvido especificamente para o contexto de exercício físico por Vlachopoulos e Michailidou (2006) e posteriormente traduzida e validada para a língua Portuguesa (Moutão, Cid, Leitão, Alves, \& Vlachopoulos, 2012) com o intuito de avaliar a perceção que os praticantes têm da satisfação das suas NPB. Este questionário é constituído por 12 itens, distribuídos pelos fatores de autonomia (e.g. faço exercício de acordo com aquilo que pretendo fazer), competência (e.g. sinto que o exercício é uma actividade que faço muito bem) e relação (e.g. tenho uma relação próxima com as pessoas com quem faço exercício). As respostas são dadas numa escala ordinal de 1-5, correspondendo a opção "Discordo Totalmente" ao valor 1 e o "Concordo Totalmente" ao valor 5. No presente estudo utilizámos um índice global de satisfação, constituído pelas três necessidades psicológicas, que foi validado num modelo de medida como fator de $2^{\text {a }}$ ordem (Moutão et al., 2012). A utilização de um índice desta natureza não é inédita e encontra suporte empírico noutros estudos (Vlachopoulos, 2007). Este índice apresentou um valor de consistência interna razoável no presente estudo (alfa de Cronbach $=0.74$ ).

Satisfaction with Life Scale - versão
Portuguesa (SWLSP): consiste num instrumento de autorrelato desenvolvido por Diener, Emmons, Larsen e Griffin, (1985), o qual foi traduzido e validado para a língua Portuguesa por Neto (1993). Este questionário é constituído é constituído por 5 itens, que concorrem para um único fator que avalia a perceção de satisfação com a vida como medida de bem-estar subjetivo (e.g. as minhas condiçôes de vida são excelentes; até ao momento tenho alcançado as coisas importantes que quero para a minha vida). A resposta é dada numa escala ordinal de 1-7, correspondendo a opção "Discordo Totalmente" ao valor 1 e o "Concordo Totalmente" ao valor 7 . No presente estudo este fator apresentou níveis elevados de consistência interna (alfa de Cronbach $=0.88$ ).

Rosenberg Self-Esteem Scale - versão Portuguesa (RSESP): consiste num instrumento de autorrelato desenvolvido por Rosenberg (1965) o qual foi traduzido e validado para a língua Portuguesa por Faria e Silva (2000). Este questionário é constituído por 10 itens, metade estão enunciados positivamente e a outra metade negativamente. A estrutura fatorial da RSES constitui um tópico que se tem revestido de alguma controvérsia (Marsh, 1996), na medida em que alguns estudos sugerem uma estrutura unidimensional enquanto outros apontam para uma organização bidimensional, com os itens enunciados positivamente separados dos enunciados negativamente em duas escalas distintas com 5 itens cada. Numa investigação constituída por três estudos Santos e Maia (2003) analisaram a estrutura fatorial da RSESp, recorrendo à análise fatorial confirmatória e concluíram que a RSESp avalia um constructo unidimensional, como sugerido originalmente por Rosenberg (1965), embora os índices de ajustamento tenham sido influenciados pelo tipo de itens que a escala utiliza (itens de orientação negativa e itens de orientação positiva). Por essa razão no presente estudo recorremos à avaliação da autoestima com recurso apenas à escala positiva da RSESp (e.g. sinto que tenho um certo número de boas qualidades; adoto uma atitude positiva para comigo). As respostas são dadas numa escala ordinal de 1-5, correspondendo a opção "Discordo Totalmente" ao valor 1 e o "Concordo Totalmente" ao valor 5. No presente estudo este fator, constituído pelos itens positivos, apresentou níveis de consistência interna bastante razoáveis (alfa de Cronbach $=$ 0.80).

Subjective Vitality Scale - versão Portuguesa (SVSp): consiste num instrumento de autorrelato desenvolvido por Ryan e Frederick (1997) e posteriormente traduzida e validada para a língua Portuguesa (Moutão, Alves, \& Cid, 2013). Este questionário é constituído é constituído por 6 itens, que concorrem para um único fator que avalia a vitalidade subjetiva como medida de bem-estar (e.g. sinto-me vivo e 
vitalizado), As respostas são dadas numa escala ordinal de 1-7, correspondendo a opção "Discordo Totalmente" ao valor 1 e o "Concordo Totalmente" ao valor 7. No presente estudo este fator apresentou níveis elevados de consistência interna (alfa de Cronbach $=0.90$ ).

\section{Procedimentos}

Em primeiro lugar, contataram-se os responsáveis administrativos dos ginásio de modo a informar sobre os propósitos do estudo e para obter a permissão para recolha da informação junto dos praticantes. Os praticantes foram abordados de forma aleatória pelos investigadores e assistentes de investigação junto da área de receção, antes da sessão de exercício e durante os dias da semana, em diversos períodos do dia (com especial incidência ao final do dia, uma vez que é neste horário que se concentram nos ginásios a maior parte dos praticantes). Após uma breve explicação sobre os objetivos do estudo, os instrumentos de avaliação foram aplicados de forma individual. Todos os participantes que concordaram fazer parte do estudo fizeram-no de forma voluntária e o consentimento informado foi obtido. Para além disso, foi garantida a confidencialidade dos dados recolhidos e assegurado que os mesmos não seriam, em momento algum, transmitidos a terceiros de forma individual. O tempo de aplicação dos questionários variou entre 15 a 20 minutos.

\section{Análise estatística}

Utilizou-se os modelos de equações estruturais (SEM), com o recurso ao software de análise de equações estruturais EQS 6.1 (Bentler, 2002), que é uma técnica multivariada que nos permite examinar simultaneamente as relações entre os constructos latentes e as variáveis de medida, bem como entre os diversos constructos do modelo (Hair, Black, Babin, \& Anderson, 2009). O método de estimação utilizado foi o da máxima verosimilhança (ML), através do teste estatístico robusto do qui-quadrado (ver Satorra \& Bentler, 1994), que corrige os valores para a não normalidade dos dados. De acordo com
Byrne (2006), se o coeficiente Mardia (ver Mardia, 1970) normalizado for superior a 5.0 é indicativo que os dados não têm uma distribuição normal multivariada, o que acontece no caso do presente estudo (kurtosis multivariada: Mardia $=71.227 ;$ Mardia normalizado $=60.946$ ) .

Assim, a análise de dados foi realizada em função das orientações/recomendações de diversos autores (e.g. Byrne, 2006; Hair et al,. 2009; Kline, 2011), que são operacionalizadas por Cid, Rosado, Alves e Leitão (2012). Para além do teste $S-B \chi^{2}$, e respetivos graus de liberdade $(d f)$ e nível de significância $(p)$, recomenda-se também a utilização de outros índices de ajustamento, considerando-se como fundamentais os seguintes indicadores de avaliação dos modelos: Standardized Root Mean Square Residual (SRMR), Comparative Fit Index (CFI), Non-Normed Fit Index (NNFI), Root Mean Square Error of Approximation (RMSEA) e o respetivo intervalo de confiança (RMSEA 90\% CI). No presente estudo, para os índices referidos, foram adotados os valores de corte sugeridos por Hu e Bentler (1999): SRMR $\leq 0.080$, CFI e NNFI $\geq 0.950$ e RMSEA $\leq 0.060$, embora no caso dos índices incrementais (CFI e $\mathrm{NNFI}$, possamos aceitar valores iguais ou superiores a 0.90 (Marsh, Hau, \& Wen, 2004).

\section{RESULTADOS}

\section{Ajustamento dos modelos de medida}

Os modelos de medida foram examinados através de análises fatoriais confirmatórias (AFC) que avaliaram o ajustamento da estrutura uni-fatorial dos questionários PASECQp, SVSp, RSESp e SWLSp, bem como da estrutura tri-fatorial da BPNESp (Tabela 1). Considerando que no presente estudo foi utilizado um índice global de satisfação, constituído pelas três NPB, bem como, um índice global de bem-estar psicológico (BEP), constituído por três indicadores (i.e. satisfação com a vida, vitalidade e autoestima), apresentamos também na mesma tabela os resultados de ajustamento destes modelos de medida testados com recurso à definição de modelos hierárquicos de fatores de $2^{\mathrm{a}}$ ordem. 
Tabela 1

Análise fatorial confirmatória e respetivos índices de ajustamento obtidos para cada um dos modelos de medida utilizados

\begin{tabular}{lccccccc}
\hline Modelos & S-B $\chi^{2}$ & $p$ & NNFI & CFI & SRMR & RMSEA & $90 \%$ IC \\
\hline PASECQp & 43.999 & 0.000 & 0.977 & 0.987 & 0.0194 & 0.068 & $0.049-0.088$ \\
BPNESp & 277.508 & 0.000 & 0.923 & 0.940 & 0.0444 & 0.067 & $0.060-0.075$ \\
RSESp & 2.580 & 0.630 & 1.000 & 1.000 & 0.0080 & 0.000 & $0.000-0.039$ \\
SVSp & 78.782 & 0.000 & 0.962 & 0.980 & 0.0232 & 0.095 & $0.076-0.114$ \\
SWLSp & 28.564 & 0.000 & 0.982 & 0.991 & 0.0186 & 0.069 & $0.046-0.095$ \\
Modelo NPB & 277.513 & 0.000 & 0.924 & 0.940 & 0.0444 & 0.066 & $0.059-0.074$ \\
Modelo BEP & 743.275 & 0.000 & 0.915 & 0.929 & 0.0522 & 0.080 & $0.075-0.086$
\end{tabular}

Nota: S-B $\chi^{2}=$ Qui-Quadrado corrigido pela formula Satorra-Bentler; $p=$ grau de significância do teste de Qui-Quadrado; $N N F I=$ Bentler-Bonnett Nonnormed Fit Index; CFI = Comparative Fit Index; SRMR - Standardized Root Mean Square Residual; RMSEA = Root Mean-Squared Error of Approximation; 90\% IC = intervalo de confiança a 90\% para RMSEA; NPB = Necessidades psicológicas básicas; $B E P=$ Bem-estar psicológico

Como se pode observar na tabela 1 , os resultados da AFC demonstram que todos os modelos de medida utilizados possuem índices de ajustamento aceitáveis.

\section{Análise descritiva, correlacional e de fiabilidade interna}

É possível verificar na Tabela 2 que para todas as variáveis latentes o coeficiente de fiabilidade interna foi acima do valor de corte 0.70, definido por Nunnally (1978). Em geral, os praticantes de fitness revelaram valores relativamente elevados de perceção de suporte de autonomia, satisfação das NPB, e de bemestar psicológico. A matriz de correlação demonstra que todas as variáveis se correlacionam positivamente de forma significativa $(p<0.01)$, em especial, entre a perceção de suporte de autonomia e a satisfação das necessidades psicológicas básicas $(r=0.55)$ e entre estas e o bem-estar psicológico ( $r=$ 0.41).

Tabela 2

Estatística descritiva, correlaçôes e consistência interna (na diagonal) das variáveis latentes presentes nos modelos de equações estruturais analisados

\begin{tabular}{lllllll}
\hline \multicolumn{1}{c}{ Fatores } & Mín - Máx & $M$ & $D P$ & \multicolumn{1}{c}{ SA } & NPB & BEP \\
\hline Suporte autonomia (SA) & $3.33-7.00$ & 5.69 & 0.82 & 0.88 & & \\
Necessidades psicológicas básicas (NPB) & $2.75-5.00$ & 4.11 & 0.43 & $0.55^{* *}$ & 0.74 & \\
Bem-estar psicológico (BEP) & $1.40-6.33$ & 4.72 & 0.70 & $0.27^{* *}$ & $0.41^{* *}$ & 0.77 \\
\hline
\end{tabular}

Nota: $M=$ Média; $D P=$ desvio-padrão; Mín. = valor mínimo; Máx. = valor máximo.

** $p<.01$

\section{Análise do efeito mediador das NPB}

Foram testados dois modelos de equações estruturais para testar o efeito mediador das NPB na relação entre o suporte de autonomia e os indicadores de bem-estar psicológico utilizados. No Modelo 1 o suporte de autonomia está definido como tendo um impacto direto sobre os indicadores de bem-estar psicológico utilizados (Figura 1). No Modelo 2 o suporte de autonomia está definido como tendo um impacto direto sobre as NPB e os indicadores de bem-estar psicológico, e as NPB definidas como tendo um impacto direto sobre os indicadores de bem-estar psicológico. Ou seja as NPB são introduzidas como mediadoras da relação suporte de autonomia $=>$ indicadores de bemestar psicológico.

Os resultados revelaram qua ambos os modelos apresentam um bom ajustamento aos dados da amostra: Modelo 1 ( $\mathrm{S}-\mathrm{B} \chi^{2}=89.806$; $p<0.000 ; \mathrm{NNFI}=.978 ; \mathrm{CFI}=0.984$; $\mathrm{SRMS}=$ 0.024; RMSEA $=0.050$; RMSEA $90 \% \mathrm{CI}=0.039$ - 0.061); Modelo 2 (S-B $\chi^{2}=199.803 ; p<0.000$; $\mathrm{NNFI}=0.963 ; \mathrm{CFI}=.971 ; \quad \mathrm{SRMS}=0.032$; RMSEA $=0.054$; RMSEA 90\% CI $=0.047$ 0.063). Da observação dos parâmetros 
individuais verifica-se que o efeito do suporte de autonomia sobre os indicadores de bem-estar psicológico foram substancialmente reduzidos no Modelo 2 quando as NPB foram introduzidas como mediadoras desta relação.

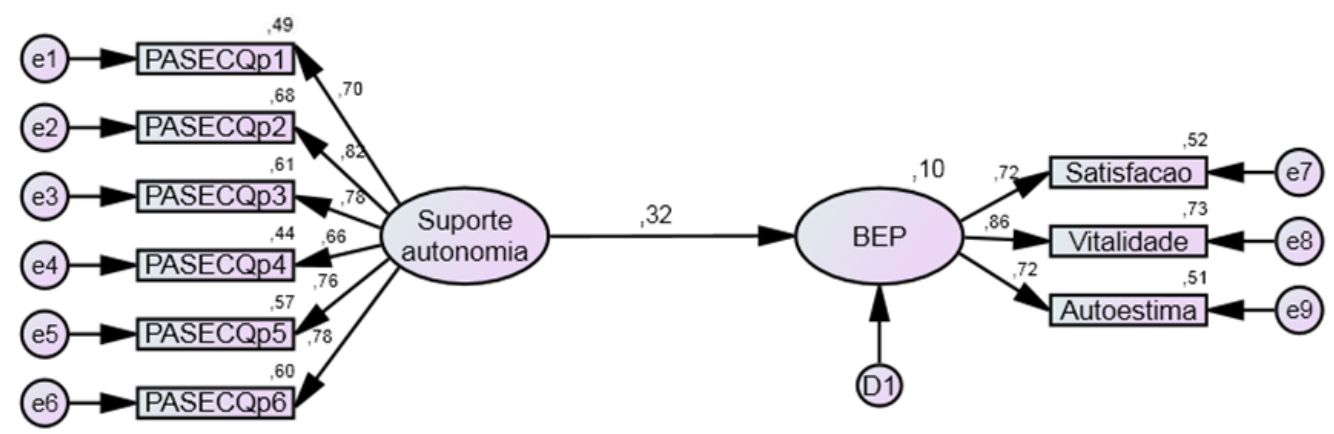

Figura 1. Modelo de equações estruturais em que se analisa o efeito direto do suporte de autonomia sobre o bem-estar psicológico (Modelo 1). Legenda: PASECQp 1-6 = Número dos itens do PASECQp; e = erro de medida para cada item; D 1 = Distúrbio ou erro de medida para o fator; $\mathrm{BEP}=$ Bem-estar psicológico; $\mathrm{NPB}=$ Necessidades psicológicas básicas

Nota: Os valores no canto superior dos retângulos representam a proporção da variância explicada (i,e, Squared Multiple Correlation); os valores nas setas de ligação representam a magnitude dos efeitos de uma variável sobre a outra; todos os parâmetros são apresentados de forma estandardizada e representam efeitos significativos $(\mathrm{p}<0.01)$

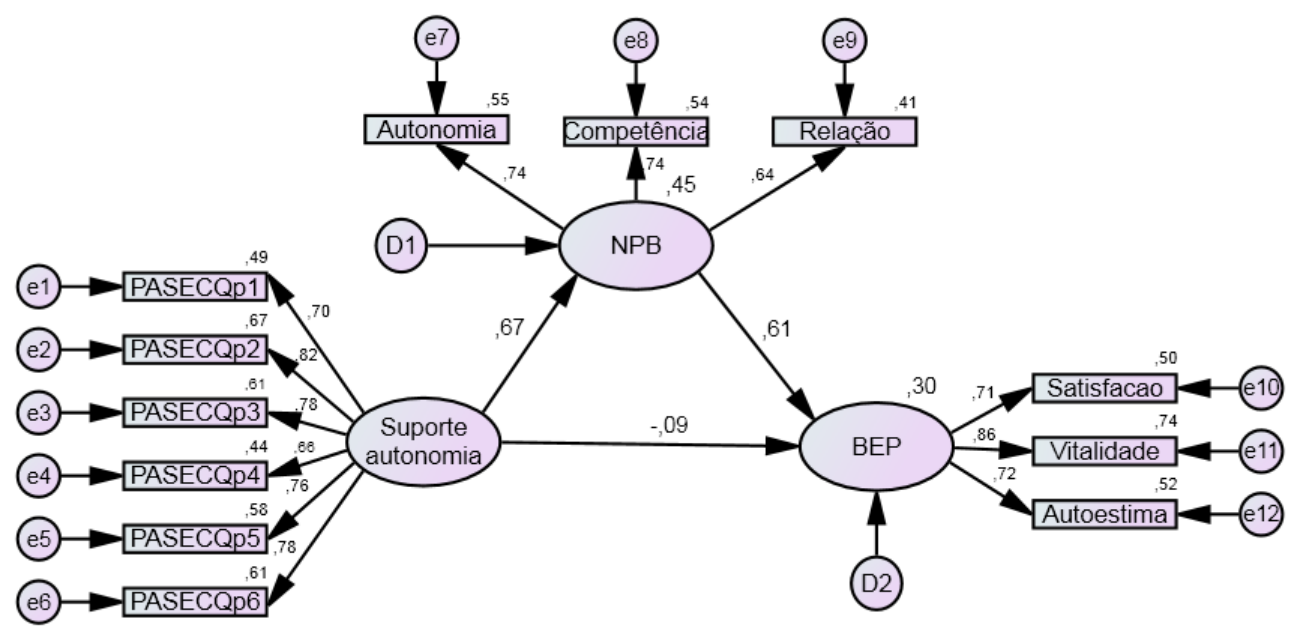

Figura 2. Modelo de equações estruturais que analisa o efeito de mediação das NPB na relação entre o suporte autonomia sobre indicadores de bem-estar psicológico (Modelo 2). Legenda, PASECQp 1-6 = Número dos itens do PASECQp; e = erro de medida para cada item; D 1-2 = Distúrbio ou erro de medida para cada fator; $\mathrm{BEP}=$ Bem-estar psicológico; NPB = Necessidades psicológicas básicas.

Nota: Os valores no canto superior dos retângulos representam a proporção da variância explicada (i,e. Squared Multiple Correlation); os valores nas setas de ligação representam a magnitude dos efeitos de uma variável sobre a outra; todos os parâmetros são apresentados de forma estandardizada e representam efeitos significativos $(\mathrm{p}<0.01)$, com exceção da ligação entre o suporte da autonomia e o BEP. 


\section{DISCUSSÃO}

O presente estudo objetivou analisar o papel mediador da perceção de satisfação das necessidades psicológicas básicas na relação entre a perceção de suporte de autonomia dado pelo instrutor de fitness e indicadores de bemestar psicológico, com recurso a análise de equações estruturais. Os resultados confirmaram o papel de mediação das NPB entre o efeito do ambiente social (i.e. suporte da autonomia dado pelos instrutores de fitness) e o bem-estar psicológico dos praticantes de fitness. Para que o efeito mediador esteja presente é necessário que se cumpram alguns critérios (ver Baron \& Kenny, 1986), conforme sintetizados por Hagger e Chatzisarantis (2008) e Hein e Hagger (2007), e que no caso do presente estudo se podem resumir da seguinte forma: 1) a variável independente (i.e. suporte de autonomia) deve estar relacionada de forma significativa com a variável dependente (i.e. bem-estar psicológico) $(\beta=0.32)$, assim como, a variável mediadora (i.e. necessidades psicológicas básicas) com a variável independente (i.e. suporte de autonomia) ( $\beta=$ $0.67)$; 2) a variável mediadora (i.e., necessidades psicológicas básicas) deve ter também um efeito direto significativo sobre a variável dependente (i.e. bem-estar psicológico) $(\beta=0.61)$; 3) e mais importante, a inclusão da variável mediadora (i.e. necessidades psicológicas básicas) como preditora da variável dependente (i.e. bem-estar psicológico) deve reduzir ou atenuar o efeito da variável independente (i.e., suporte de autonomia) sobre a dependente (i.e., bem-estar psicológico) (o efeito direto passou de $\beta=0.32$ para $\beta=$ 0.09 - valor este não significativo). Para além disso, segundo Hair et al. (2009), também podemos confirmar se o efeito da mediação está presente analisando os efeitos indiretos entre os parâmetros. Se o efeito indireto entre a variável independente (i.e. suporte de autonomia) e a dependente (i.e. bem-estar psicológico) é significativo $(\beta=0.41)$ através da variável mediadora (i.e. necessidades psicológicas básicas), enquanto que o efeito direto não é significativo $(\beta=-0.09)$, então estamos perante um efeito de mediação, que segundo Baron e
Kenny (1986) se refere à transferência do efeito de uma variável (independente/preditora: suporte de autonomia) sobre outra (dependente/resultado: bem-estar psicológico) para uma terceira variável mediadora (i.e. necessidades psicológicas básicas).

Estes resultados suportam empiricamente o mecanismo de mediação através do qual o efeito do suporte de autonomia se faz sentir sobre o bem-estar psicológico, ou seja, através da satisfação das NPB, conforme sugerido pela SDT (Deci \& Ryan, 1985, 2008) e pelo modelo hierárquico de motivação intrínseca-extrínseca proposto por Vallerand (1997). Os resultados vão também ao encontro do estudo realizado por Vlachopoulos e Karavani (2009), cujos resultados evidenciam igualmente este efeito mediador das NPB entre o suporte de autonomia e a vitalidade subjetiva.

Este mecanismo pode assim explicar os resultados obtidos nalguns estudos realizados com o propósito de testar os efeitos de alguns fatores do ambiente social sobre a resposta afetiva ao exercício físico, designadamente do estudo realizado por Turner, Rejeski, e Brawley (1997) onde se verificou que, após uma série intensa de exercícios, os praticantes que receberam mais feedback positivo e experienciaram uma atmosfera social mais rica reportaram um aumento de revitalização comparativamente aos praticantes que foram orientados por um instrutor com um comportamento neutro.

De forma similar, no estudo realizado por Fox, Rejeski, e Gauvin (2000) verificou-se que os praticantes envolvidos numa classe de exercício num ambiente socialmente mais rico proporcionado pelo instrutor e pelos colegas, reportaram valores mais elevados de divertimento e prazer após a realização do exercício comparativamente aos praticantes que realizaram os mesmos exercícios num ambiente socialmente neutro.

Gostaríamos ainda de referir, que existem algumas limitações no presente estudo que importa indicar. O efeito de mediação das NPB foi analisado através de equações estruturais, com recurso a uma amostra transversal de dados, seguindo os mesmos procedimentos 
adotados noutros estudos (e.g. Gunnell, Mack, Wilson, \& Adachi, 2011; López-Walle, Balaguer, Castillo, \& Tristán, 2012), considerando a existência prévia de um conjunto consistente de estudos que comprovam a existência de uma relação de causa efeito e de a teoria subjacente sugerir direções inferenciais que são inerentemente causais. Apesar de os estudos de mediação com recurso a amostras transversais tenderem a ser os mais comummente reportados em estudos empíricos de mediação (Rose, Holmbeck, Coakley \& Franks, 2004), alguns autores sugerem que para que o padrão temporal inerente possa ser captado devem também ser realizados ensaios clínicos controlados randomizados (Kraemer, Wilson, Fairburn, \& Agras, 2002; Selig \& Preacher, 2009). Investigação futura com desenhos experimentais deverá ser desenvolvida para examinar de que forma a mudança ao longo do tempo do clima motivacional de suporte á autonomia proporcionado pelo instrutor vai afetando o efeito de mediação das NPB sobre o bem-estar de praticantes de exercício.

Por outro lado, na presente investigação foi também objetivada a análise do efeito direto da satisfação das NPB sobre o bem-estar, conforme vem recentemente a ser demonstrada (Gunnell, Crocker, Wilson, Mack, \& Zumbo, 2013; Gunnell et al., 2011; Ng, Ntoumanis, Thøgersen-Ntoumani, Stott, \& Hindle, 2013; Sylvester, Mack, Busseri, Wilson, \& Beauchamp, 2012). Contudo, será também importante de futuro considerar a inclusão de outras variáveis que não foram incluídas no modelo, como o caso das regulações comportamentais, as quais poderão ter um papel importante também na mediação do efeito da satisfação das NPB sobre o bem-estar (Teixeira, Patrick, \& Mata, 2011).

Por último, tendo em conta que os participantes deste estudo posicionaram-se em função do suporte de autonomia que percecionam por parte do instrutor com quem estão mais tempo, será também importante nestes estudos futuros analisar os efeitos à exposição de instrutores com diferentes estilos de suporte de autonomia, incluindo também o efeito da exposição a outros significativos (e.g. colegas de treino, staff), como habitualmente acontece durante a prática de exercício em ginásios.

\section{CONCLUSÕES}

Dada a importância patenteada nesta investigação sobre o papel mediador da satisfação das NPB na promoção do bem-estar psicológico, será importante que os técnicos de exercício físico, bem como, todos os agentes que interagem neste contexto, olhem para $o$ exercício físico como algo mais do que mero movimento potenciador de gasto calórico. $\mathrm{Na}$ nossa opinião, é fundamental que olhem para exercício e os seus praticantes numa perspetiva mais holística, tomando em consideração as contribuições de diferentes áreas disciplinares na construção dos programas de exercício. A promoção da prática de exercício com vista à melhoria da saúde, não se pode centrar apenas na mensagem de desenvolvimento da aptidão/condição física, no "gasto calórico" e nos aspetos relacionados com a saúde física, mas também na valorização das dimensões de bem-estar e qualidade de vida (Varo et al., 2003). A intervenção dos técnicos de exercício deve ir ao encontro daquilo que as pessoas esperam alcançar quando fazem exercício: "feelgood factor" (Biddle \& Ekkekakis, 2005, p. 141), o que realça uma perspetiva mais humanista da prática. Este novo paradigma apela a que os ginásios e health clubs se reinventem e reflitam em novos modelos de intervenção, onde a satisfação das NPB seja uma preocupação central, com vista à melhoria do bem-estar dos praticantes de fitness. Esta questão passa pelo desenvolvimento de programas de exercício inovadores, não monótonos, que: a) promovam a autonomia dos praticantes, envolvendo-os no processo de decisão; b) aumentem a sua perceção de competência, proporcionando situações de sucesso; c) e, ao mesmo tempo, promovam o estabelecimento e fortalecimento de relações interpessoais positivas. 


\author{
Agradecimentos: \\ Nada a declarar
}

\section{Conflito de Interesses:}

Nada a declarar.

Financiamento:

Nada a declarar.

\section{REFERÊNCIAS}

Baron, R., \& Kenny, D. (1986). The moderatormediator variable distinction in social psychology research: Conceptual, strategic, and statistical considerations. Journal of Personality and Social Psychology, 51(6), 1173-1182. doi: 10.1037//0022-3514.51.6.1173

Bentler, P. (2002). EQS 6 Structural equations program manual. Encino, CA: Multivariate Software, Inc.

Biddle, S., \& Ekkekakis, P. (2005). Physically active lifestyles and well-being. In F. Huppert, N. Baylis \& B. Keverne (Eds.), The Science of WellBeing (pp. 141-168). Oxford: Oxford University Press.

Byrne, B. (2006). Structural equation modeling with EQS: Basic concepts, applications, and programming (2nd ed.). Mahwah, NJ: Lawrence Erlbaum Associates.

Deci, E. L., Eghrari, H., Patrick, B. C., \& Leone, D. R. (1994). Facilitating internalization: The selfdetermination theory perspective. Journal of Personality, 62(1), 119-142. doi: 10.1111/j.1467-6494.1994.tb00797.x

Deci, E. L., \& Ryan, R. M. (1985). Intrinsic motivation and self-determination in human behavior. New York: Plenum Press.

Deci, E. L., \& Ryan, R. M. (2008). Self-determination theory: A macrotheory of human motivation, development, and health. Canadian Psychology, 49(3), 182-185. doi: 10.1037/a0012801

Diener, E., Emmons, R. A., Larsen, R. J., \& Griffin, S. (1985). The satisfaction with life scale. Journal of Personality Assessment, 49(1), 71-75. doi: 10.1207/s15327752jpa4901_13

Cid, L., Rosado, A., Leitão, C., \& Alves, J. (2012). Tradução e Validação de Questionários em Psicologia do Desporto. In António Rosado, Isabel Mesquita \& Carlos Colaço (Eds.), Métodos e Técnicas de Investigação Qualitativa. Lisboa: Edições FMH.

Edmunds, J., Duda, J. L., \& Ntoumanis, N. (2008). Testing a self-determination theory-based teaching style intervention in the exercise domain. European Journal of Social Psychology, 38(2), 375-388. doi: 10.1002/ejsp.463
Edmunds, J., Ntoumanis, N., \& Duda, J. (2006). A test of self-determination theory in the exercise domain. Journal of Applied Social Psychology, 36(9), 2240-2265. doi: 10.1111/j.00219029.2006.00102.x

Faria, L., \& Silva, S. (2000). Efeitos do exercício físico na promoção do auto-conceito. Psychologica, 25, 25-43.

Fox, L. D., Rejeski, W. A., \& Gauvin, L. (2000). Effects of leadership style and group dynamics on enjoyment of physical activity. American Journal of Health Promotion, 14(5), 277-283. doi: 10.4278/0890-1171-14.5.277

Gunnell, K. E., Crocker, P. R. E., Wilson, P. M., Mack, D. E., \& Zumbo, B. D. (2013). Psychological need satisfaction and thwarting: A test of basic psychological needs theory in physical activity contexts. Psychology of Sport and Exercise, 14, 599-607.

doi: 10.1016/j.psychsport.2013.03.007

Gunnell, K. E., Mack, D. E., Wilson, P. M., \& Adachi, J. (2011). Psychological needs as mediators? The relationship between leisure time physical activity and well being in people diagnosed with osteoporosis. Research Quarterly for Exercise \& Sport, 82(4), 794-798.

Hagger, M., \& Chatzisarantis, N. (2008). Selfdetermination theory and the psychology of exercise. International Review of Sport and Exercise Psychology, 1(1), 79-103. doi: 10.1080/17509840701827437

Hair, J. F., Black, W. C., Babin, B. J., \& Anderson, R. E. (2009). Multivariate data analysis (7th ed.). New Jersey: Prentice Hall.

Hein, V., \& Hagger, M. S. (2007). Global self-esteem, goal achievement orientations, and selfdetermined behavioural regulations in a physical education setting. Journal of Sports Sciences, 25(2), 149 - 159. doi: $10.1080 / 02640410600598315$

Hu, L., \& Bentler, P. (1999). Cutoff criteria for fit indexes in covariance structure analysis: conventional criteria versus new alternatives. Structural Equation Modeling, 6(1), 1-55. doi: 10.1080/10705519909540118

López-Walle, J., Balaguer, I., Castillo, I., \& Tristán, J. (2012). Autonomy Support, Basic Psychological Needs and Well-Being in Mexican Athletes. The Spanish Journal of Psychology, 3(15), 12831292.

doi.org/10.5209/rev_SJOP.2012.v15.n3.39414

Kiviniemi, M. T., Voss-Humke, A. M., \& Seifert, A. L. (2007). How do I feel about the behaviour? The interplay of affective associations with behaviors and cognitive beliefs as influences on physical activity behaviour. Health Psychology, 26(2), 152-158. doi: 10.1037/02786133.26 .2 .152

Kline, R. (2011). Principles and Practice of Structural Equation Modeling ( $3^{\text {nd }}$ ed.). New York: The Guilford Press. 
Kraemer, H. C., Wilson, G. T., Fairburn, C. G., \& Agras, W. S. (2002). Mediators and moderators of treatment effects in randomized clinical trials. Arch Gen Psychiatry, 59(10), 877-883.

Mardia, K. (1970). Measures of multivariate skewness and kurtosis with applications. Biometrika, 57(3), 519-530. doi: $10.2307 / 2334770$

Marsh, H. W. (1996). Positive and negative global self-esteem: A substantively meaningful distinction or artifactors? Jounal of Personality and Social Psychology, 70(4), 818-819. doi: 10.1037//0022-3514.70.4.810

Marsh, H. W., Hau, K. T., \& Wen, Z. (2004). In search of golden rules: Comment on hypothesis-testing approaches to setting cutoff values for fit indexes and dangers in overgeneralizing $\mathrm{Hu} \&$ Bentler's (1999) findings. Structural Equation Modeling, 11(3), 320-341.

Moutão, J., Alves, S., \& Cid, L. (2013). Traducción y validación de la Subjective Vitality Scale en una muestra de practicantes de ejercicio portugueses. Revista Latinoamericana de Psicologia, 45(2), 223-230.

Moutão, J., Cid, L., Leitão, J. C., \& Alves, J. (2012). Tradução e validação inicial do questionário Perceived Autonomy Support: Exercise Climate Questionnaire (PASECQp) numa amostra de praticantes de exercício portugueses. Psicologia: Reflexão e Critica, 25(4), 701-708. doi: 10.1590/S0102-79722012000400009

Moutão, J., Cid, L., Leitão, J. C., Alves, J., \& Vlachopoulos, S. P. (2012). Validation of the Basic Psychological Needs in Exercise Scale in a Portuguese Sample. Spanish Journal of Psychology, 15(1), 399-409. doi: 10.5209/rev_SJOP.2012.v15.n1.37346

Neto, F. (1993). The satisfaction with life scale: Psychometric properties in an adolescent sample. Journal of Youth and Adolescence, 22(2), 125-134. doi: 10.1007/BF01536648

Ng, J., Ntoumanis, N., Thøgersen-Ntoumani, C., Stott, K., \& Hindle, L. (2013). Predicting psychological needs and well-being of individuals engaging in weight management: The role of important others. Applied Psychology: Health and Well-Being, 5(3), 291310. doi: 10.1111/aphw.12011

Nunnally, J. C. (1978). Psychometric theory. New York: McGraw-Hill Inc.

Rose, B. M., Holmbeck, G. N., Coakley, R. M., \& Franks, E. A. (2004). Mediator and moderator effects in developmental and behavioral pediatric research. Developmental and Behavioral Pediatrics, 25(1), 58-67.

Rosenberg, M. (1965). Society and the adolescent self-image. Princeton: Princeton University Press.

Ryan, R. M. (1995). Psychological needs and the facilitation of integrative processes. Journal of
Personality, 63(3), 397-427. doi: 10.1111/j.1467-6494.1995.tb00501.x

Ryan, R. M., \& Frederick, C. (1997). On energy, personality, and health: subjective vitality as a dynamic reflection of well-being. Journal of Personality, 6(3), 529-565. doi: 10.1111/j.1467-6494.1997.tb00326.x

Santos, P. J., \& Maia, J. (2003). Análise factorial confirmatória e validação preliminar de uma versão portuguesa da Escala de Auto-Estima de Rosenberg. Psicologia: Teoria, Investigação e Prática, 8, 253-268.

Satorra, A., \& Bentler, P. (1994). Corrections to test statistics and standard errors in covariance structure analysis. In A. Eye \& C. Clogg (Eds.), Latent variables analysis: Applications for development research (pp. 399-419). Thousand Oaks, California: Sage Publications.

Selig, J. P., \& Preacher, K. J. (2009). Mediation models for longitudinal data in developmental research. Research in Human Development, 6(2-3), 144-164. doi: 10.1080/15427600902911247

Silva, M. N., Markland, D., Carraça, E., Vieira, P. N., Coutinho, S. R., Minderico, C. S., Matos, M. G., Sardinha, L. B., \& Teixeira, P. J. (2011). Exercise autonomous motivation predicts 3year weight loss in women. Medicine and Science in Sports and Exercise, 43(4), 728-737. doi: 10.1249/MSS.0b013e3181f3818f

Silva, M. N., Markland, D., Vieira, P. N., Coutinho, S. R., Palmeira, A. L., Carraça, E. V., Minderico, C. S., Matos, M. G., Sardinha, L. B., \& Teixeira, P. J. (2010). Helping overweight women become more active: need support and motivational regulations for different forms of physical activity. Psychology of Sport and Exercise, 11(6), 591-601. doi: 10.1016/j.psychsport.2010.06.011

Sylvester, B. D., Mack, D. E., Busseri, M. A., Wilson, P. M., \& Beauchamp, M. R. (2012). Health enhancing physical activity, psychological need satisfaction, and well-being: Is it how often, how long, or how much effort that matters? Mental Health \& Physical Activity, 5(2), 141147. doi: doi:10.1016/j.mhpa.2012.10.004

Teixeira, P. J., Carraça, E. V., Markland, D., Silva, M. N., \& Ryan, R. M. (2012). Exercise, physical activity, and self-determination theory: A systematic review. International Journal of Behavioral Nutrition and Physical Activity, 9, 78. doi: 10.1186/1479-5868-9-78

Teixeira, P. J., Silva, M. N., Coutinho, S. R., Palmeira, A. L., Mata, J., Vieira, P. N., Carraça, E. V., Santos, T. C., \& Sardinha, L. B. (2010). Mediators of weight loss and weight loss maintenance in middle-aged women. Obesity, 18(4), 725-735. doi: 10.1038/oby.2009.281

Turner, E. E., Rejeski, W. J., \& Brawley, L. R. (1997). Psychological benefits of physical activity are influenced by the social environment. Journal of Sport \& Exercise Psychology, 19(2), 119-130. 
Vallerand, R. J. (1997). Toward a hierarchical model of intrinsic and extrinsic motivation. Advances in Experimental Social Psychology, 29, 271-360. doi.org/10.1016/S0065-2601(08)60019-2

Varo, J., Martinez-Gonzalez, M., De Irala-Estévez, J., Kearney, J., Gibney, M., \& Martínez, J. A. (2003). Distribution and determinants of sedentary lifestyles in the European Union. International Journal of Epidemiology, 32(1), 138-146. doi: 10.1093/ije/dyg116

Vieira, P. N., Mata, J., Silva, M. N., Coutinho, S. R., Santos, T. C., Minderico, C. S., Sardinha, L. B., \& Teixeira, P. J. (2011). Predictors of psychological well-being during behavioral obesity treatment in women. Journal of Obesity, 1-8. doi: 10.1155/2011/936153

Vieira, P. N., Silva, M. N., Mata, J., Coutinho, S. R., Santos, T. C., Sardinha, L. B., \& Teixeira, P. J. (2013). Correlates of health-related quality of life, psychological well-being, and eating selfregulation after successful weight loss maintenance. Journal of Behavioral Medicine, 36(6), 601-610. doi.org/10.1007/s10865-0129454-9

Vlachopoulos, S. (2007). Psychometric evaluation of the basic psychological needs in exercise scale in community exercise programs: A crossvalidation approach. Hellenic Journal of Psychology, 4, 52-74.

Vlachopoulos, S., \& Karavani, E. (2009). Psychological needs and subjective vitality in exercise: a cross-gender situational test of needs universality hypothesis. Hellenic Journal of Psychology, 6, 207-222.

Vlachopoulos, S., \& Triantafyllidou, O. (2008). The contribution of psychological need satisfaction to the prediction of exercise-induced feeling states. Hellenic Journal of Physical Education and Sport Science, 28(4), 10-31.

Vlachopoulos, S. P., \& Michailidou, S. (2006). Development and initial validation of a measure of autonomy, competence and relatedness in exercise: The basic psychological needs in exercise scale. Measurement in physical education and exercise science, 10(3), 179-201. doi: 10.1207/s15327841mpee1003_4

Williams, D. M., Dunsiger, S., Ciccoli, J. T., Lewis, B. A., Albrecht, A. E., \& Marcus, B. H. (2008). Acute affective response to a moderateintensity exercise stimulus predicts physical activity participation 6 and 12 months later. Psychology of sport and exercise, 9(3), 231-245. doi: 10.1016/j.psychsport.2007.04.002

Williams, G. C., Grow, V. M., Freedman, Z. R., Ryan, R. M., \& Deci, E. L. (1996). Motivational predictors of weight loss and weight-loss maintenance. Journal of Personality and Social Psychology, 70(1), 115-126. doi: 10.1037//0022-3514.70.1.115

Teixeira, P. J., Patrick, H., \& Mata, J. (2011). Why we eat what we eat: the role of autonomous motivation in eating behaviour regulation. Nutrition Bulletin, 36, 102-107. 